\title{
Analysis of photonic crystal fibers using full vectorial finite difference method
}

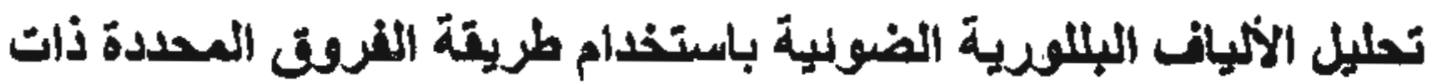

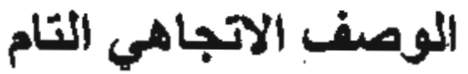

\author{
M.Farhat" ${ }^{\prime *}$ S.S.A.Obayya ${ }^{2}$ and A.M.Nasr ${ }^{\text {** }}$ \\ 'Department of Mathematical and Physical Sciences. Faculty of Engineering. \\ University of Mansoura, Egypt. \\ Email: "engmfarhat@mans.edu.eg \\ *. fldp7@mans.edu.eg \\ 'Department of Electronics and Communications. Faculty of Engineering. \\ University of Mansoura. Egypl
}

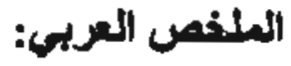

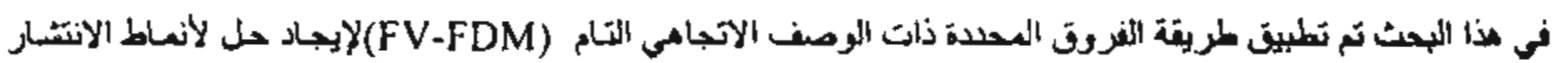

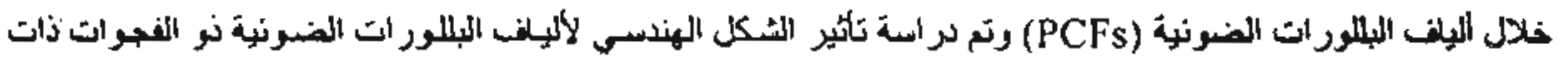



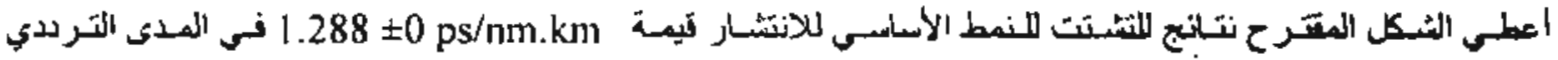

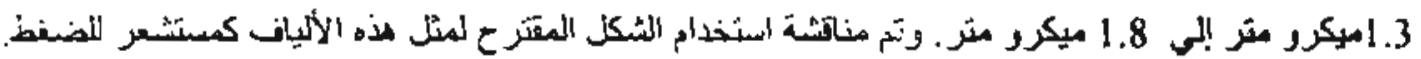

\begin{abstract}
Abst ract
The full-vectorial finite difference method (FV-FDM) is applied to perform modal solution to photonic crystal fibers (PCFs). The effects of geometrical parameters of the circular and elliptical holes on the effective index. the dispersion and the effective mode area of the fundamental mode have been studied. PCF structure showing dispersion of $0 \pm 1.288 \mathrm{ps} / \mathrm{nm} . \mathrm{km}$ over the wavelenglh range from $1.3 \mu \mathrm{m}$ to $1.8 \mu \mathrm{m}$ has been reported. The applicability of using PCF as a pressure sensor has been discussed.
\end{abstract}

Keywords: Photonic crysta! fiber: Finite difference method: Full-vectorial modes

Accepted May 29, 2007 


\section{1- Introduction}

Photonic Crystal Fibers (PCFs) [1.2] have been the subject of extensive research in recent years. This due to their unusual and very attractive optical properties such as a wide single mode wavelength range [3]. large effective mode area [4] and anomalous dispersion at visible and near infrared wavelengths $[5]$ : They are usually made of silica with a regular array of air holes running along the length of the fiber acting as a cladding. Such structure creates bandgaps where propagation at certain optical frequencies is forbidden. A defect is introduced in the periodic structure in the form of either larger air hole (low index core), or missing hole (high index core) which introduces guided modes within the previously forbidden bandgap.

Intensive research: has .been ongoing during the last years to develop accurate modeling methods for photonic crystal. Various methods have been developed for modal calculation of such waveguides such as finite difference time domain [6]. Linite difference method [7] and the tinite element method [8,9]. Due to the simpliçity of its implementation and the sparsity of its resultant matrix, mode solvers based on the FDM have become attraetive numierical methods for analyzing the propagation characteristics of the optical or dielectric waveguides. especially for those complex dielectric waveguides without analytical solutions. The FDM was first employed to solve the scalar waveguide modes under the weakly guiding approximation [10]. For strongly guiding structures, semi-vectorial equations for optical waveguides with arbitrary index profiles were derived [11, 12]. To obtain more accurate mode fields. a full-vectorial finite difference scheme was then proposed [13-15].

In this paper, the full vectorial mode calculation using finite-difference method (FV-FDM) [15] is used to perform modal analysis of PCFs. The effects of geometrical parameters of the circular and elliptical holes of the PCF on the effective index, the effective mode area and the dispersion of the fundamental mode have been studied thoroughly. PCFs with circular and elliptical holes with low index circular and elliptical core have been also studied. Possibility of using PCFs in 
pressure sensing has been discussed.

following this introduction. a brief mathematical analysis of the FV-FDM is introduced in section 2. The validation and simulation results of the modal analysis program are detailed in section 3 by addressing a standard rib waveguide and different PCFs structures. Finally. conclusions are drawn.

\section{2- Mathematical analysis.}

The full-rectorial ware equations based on electric fields can be derived from the Maxwell's equations [16]:

$$
\begin{aligned}
& \nabla \times E=-j(1) \mu_{0} H \\
& \nabla \times H=j \omega n^{2} \varepsilon_{\mathrm{a}} E \\
& \nabla \cdot\left(n^{2} E\right)=0 \\
& \nabla \cdot(H)=0
\end{aligned}
$$

First by taking the curl of Eq. (I) and substituting using equation Eq. (2):

$$
\nabla \times \nabla \times E-n \cdot{ }^{*} \cdot \dot{E}=0
$$

where $k=(1) / c$ is the free space wave number and $c=1 / \sqrt{\mu_{0} \varepsilon_{0}}$ is the velocity of light in free space.

By using the vector identity

$$
\nabla \times \nabla \times=\nabla(\nabla .) \cdot \nabla^{2}
$$

Eq. (5) becomes $\nabla^{2} \mathrm{E}+\Pi^{2} k^{2} \mathrm{E}=\nabla(\nabla . E)$

If the transverse components of an electromagnetic field are known. then the longitudinal component may be readily obtained by applying Eq. (3). Therefore. the transverse components are sufficient to describe the vectorial properties of the electromagnetic field. The transverse component of Eq. (7):

$$
\nabla^{2} E_{1}+n^{2} k^{2} E_{t}=\nabla_{1}\left(\nabla_{1} . E_{1}+\frac{\partial E_{2}}{\partial z}\right)
$$

and using Eq. (3):

$$
\nabla_{1},\left(\mathrm{n}^{2} \mathrm{E}_{1}\right)+\frac{\partial \mathrm{n}^{2}}{\partial \mathrm{z}} \mathrm{E}_{\mathrm{x}}+\mathrm{n}^{2} \frac{\partial \mathrm{E}_{\mathrm{x}}}{\partial \mathrm{z}}=0
$$

Since $n(x, y)$ is $z$-invariant. $\partial n^{2} / \partial z=0$. and so the longitudinal and transverse components are related by

$$
\frac{\partial E_{z}}{\partial z}=-\frac{1}{n^{2}} \nabla_{1} \cdot\left(n^{2} E_{1}\right)
$$

Substituting Eq. (10) into Eq. (8), and using the transformation

$E(x, y, z)=E(x, y) e^{-x / 2 z}$

one can derive the vectorial wave equation

$$
\begin{aligned}
& \nabla_{1}^{2} E_{1}+\left(n^{2} k^{2}-\beta^{2}\right) E_{1}= \\
& \nabla_{1}\left[\nabla_{1} \cdot E_{1}-\frac{1}{n^{2}} \nabla_{1} \cdot\left(n^{2} E_{1}\right)\right]
\end{aligned}
$$

Eq. (12) can be written in matrix fom [16]

$$
\left(\begin{array}{ll}
P_{u} & P_{x y} \\
P_{y} & P_{y y}
\end{array}\right)\left(\begin{array}{l}
E_{q} \\
E_{y}
\end{array}\right)=\beta^{2}\left(\begin{array}{l}
E_{x} \\
E_{y}
\end{array}\right)
$$


P. 4 M.Farhat. S.S.A.Obay'yan"M.M.Nasr

where the ditterential opsators are grid points. The subscripts N. S. E and W. defined as

$P_{\mathrm{u}} E_{\mathrm{r}}=\frac{\partial}{\partial \mathrm{x}}\left[\frac{1}{n^{2}} \frac{\partial\left(n^{2} E_{\mathrm{V}}\right)}{\partial \mathrm{x}}\right]+\frac{\partial^{2} E_{\mathrm{q}}}{\partial \mathrm{y}^{2}}+n^{2} \mathrm{k}^{2} E_{\mathrm{v}}$

$P_{n} E_{n}=\frac{\partial I^{2}}{\partial x^{2}}+\frac{\partial}{r}\left|\frac{1}{n^{2}} \frac{\partial(n+1)}{\partial y}\right|+n^{2} k^{2} E_{2}$

$P_{w} E_{y}=\frac{\partial}{\partial x}\left[\frac{1}{n^{2}} \frac{\partial\left(n^{2} E_{3}\right)}{\partial y}\right]-\frac{\partial^{2} E_{3}}{\partial x \partial y}$

$\left.P_{3} E_{、}=\left.\frac{\partial}{\partial}\right|_{n^{2}} \frac{1}{\partial x}\right]-\frac{\partial^{2} E_{2}}{\partial y \partial x}$

Eq. (13) is a full-vector eigenvalue equation. which describes the modes of propigation lior am incegraled wareguide The wo coupled transierse field componenls $l$, and $t_{\mathrm{y}}$ laken logether are the eigenfunction. and the corresponding eigenvalue is $\beta^{2}$. The four remaining field components can be easily derived from these two lransverse components by applying Maxwell's cquations. The field is assumed >ero at grid points immediately outside of the computation window.

In order to translate this partial differential equation into a set of finite difference equations. the sccond derivatives must be approximated in terms of the values of the fieids at surrounding

One of the most straightforward techniques for deriving finite difference approximations is Lagrange interpolation [17]. The Lagrange interpolant is simply the lowest order polynomial which goes through all of the sample points. The derivatives can then be easily computed from the polynomial coefficients of the interpolating function. For example, to 
Mansoura lingineering Journal. (ME.3), Vol. 32. No. 2. June 2007

approximate the second derivalive of $\phi$ with respect to $\mathrm{x}$ at point $P . \partial^{2} \phi /\left.\partial \mathrm{x}^{2}\right|_{\mathrm{p}}$, a quadratic equation was fitted to the three points $\phi_{1} . \phi_{1}$. and $\phi_{w}$ :

$\phi(x)= \begin{cases}A_{w}+B x+C x^{2} & \text { in cell } W \\ A_{P}+B x+C x^{2} & \text { in cell } P \\ A_{t}+B x+C x^{2} & \text { in cell } E\end{cases}$

and the interpolating function passes through the three points $e_{\mathrm{c}} \mathrm{x} \cdot \mathrm{e}_{\mathrm{v}}$, and $\mathrm{e}_{\mathrm{v}}$ :

ㄴ, $\cdots A_{11} \quad 131 \mathrm{x} \cdot(\mathrm{CW})^{?}$

$\mathrm{e}_{\mathrm{V}} \mathrm{P}=\Lambda_{\mathrm{l}}$

$e_{\mathrm{vE}}=A_{\mathrm{l}}+\mathrm{B} \Delta \mathrm{X}+\mathrm{C}(\Delta \mathrm{x})^{2}$

Moreover. with adding additional constraint that describes the continuity of $\mathrm{n}^{2} \mathrm{e}, \mathrm{at} \mathrm{x}= \pm-\mathrm{w} / 2$. one cinn get:

$$
\begin{aligned}
\because:\left\{A_{n}\right. & \left.-\frac{1}{2} B A x+\frac{1}{4} C \Delta x^{2}\right\}= \\
& \because{ }^{*}\left\{A_{p}-\frac{1}{2} B \Delta x+\frac{1}{4} C \Delta x^{2}\right\}
\end{aligned}
$$

$$
\begin{gathered}
\pi_{p}^{2}\left\{A_{p}+\frac{1}{2} B \Delta x+\frac{1}{4} C \Delta x^{2}\right\}= \\
n_{L}^{2}\left\{A_{L}+\frac{1}{2} B \Delta x+\frac{1}{4} C \Delta x^{2}\right\}
\end{gathered}
$$

From Eq. (23) to Eq. (27) give five linear equations. which can be solved for the live unknown polynomial coellicients. (Actually: $B$ and $C$ are the only two coefficients of interest because they describe respectively the first and second derivatives of e) Atter some algebraic steps. onc can get:

$$
\begin{aligned}
& \frac{\partial \phi}{\partial \mathrm{x}}=\mathrm{B}
\end{aligned}
$$

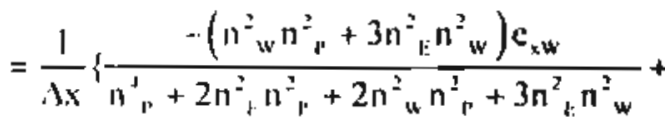

$$
\begin{aligned}
& \frac{3\left(n_{1}{ }^{2}-n^{2}{ }_{W}\right) n^{2}{ }_{{ }_{1}}}{\pi^{4}{ }_{P}+2 n^{2}{ }_{E} n^{2}{ }_{P}+2 n^{2}{ }_{W} n^{2}{ }_{P}+3 n_{E}^{2} n^{2}{ }_{W}} e_{{ }_{W}}+ \\
& \left.\frac{\left(n_{k}{ }_{k} n^{2}+3 n^{2}{ }_{E} n^{2}{ }_{W}\right)}{n^{4}{ }_{p}+2 n^{2}{ }_{E} n^{2}{ }_{P}+2 n^{2}{ }_{W} n^{2}{ }_{P}+3 n_{E}^{2} n^{2}{ }_{W}} e_{k E}\right)
\end{aligned}
$$

$$
\begin{aligned}
& \frac{\partial i d}{\partial x^{2}}=2 C
\end{aligned}
$$

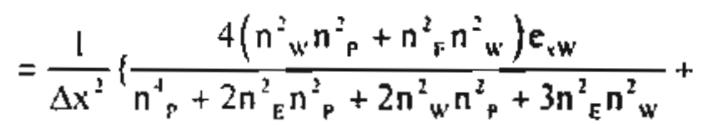

$$
\begin{aligned}
& \frac{4\left(n^{2}{ }_{1:} n_{p}{ }_{p}-n^{2}{ }_{W} n^{2}{ }_{p}+2 n^{2}{ }_{E} n^{2}{ }_{W}\right) n^{2}{ }_{p} e_{x p}}{n^{4}{ }_{p}+2 n^{2}{ }_{1:} n^{2}{ }_{p}+2 n^{2}{ }_{w} n^{2}{ }_{p}+3 n^{2}{ }_{E} n^{2}{ }_{w}}+ \\
& \left.\frac{4\left(n_{E}{ }_{E} n_{p}^{2}+n^{2}{ }_{E} n^{2}{ }_{W}\right) n^{2}{ }_{p}}{n^{4}{ }_{p}+2 n^{2}{ }_{t} n^{2}{ }_{F}+2 n^{2}{ }_{W} n^{2}{ }_{W}+3 n^{2}{ }_{E} n^{2}{ }_{W}} e_{V k}\right)
\end{aligned}
$$

Equations (18). (20) and (29). can be combined to yield the linite difference representation of the operator $P_{x x}$

\begin{tabular}{|c|c|c|}
\hline 0 & $\frac{1}{(\Delta y)^{2}}$ & 0 \\
\hline$\frac{\alpha_{w}}{(\Delta x)^{2}}$ & $n^{2}{ }_{,} k^{2}-\frac{2}{(\Delta y)^{2}}-\frac{2 \alpha_{\mathrm{P}}}{(\Delta x)^{2}}$ & $\frac{\alpha_{\mathrm{E}}}{(\Delta x)^{2}}$ \\
\hline 0 & $\frac{1}{(\Delta y)^{2}}$ & 0 \\
\hline
\end{tabular}

Where $\alpha_{\mathrm{W}}, \alpha_{\mu}$ and $\alpha_{k}$ are dimensionless ratios defined: 
$u_{n}=\frac{4\left(n^{2}{ }_{W} n^{2}{ }_{n}+n^{2}{ }_{E} n^{2}{ }_{W}\right)}{n^{1}{ }_{11}-21_{1}^{2} n^{2}{ }_{n}+2 n^{2}{ }_{n} n^{2}{ }_{n}+3 n^{2}{ }_{1} n^{2}{ }_{n}}$



$\alpha_{E} \equiv \frac{4\left(n_{E}{ }_{E} n_{p}+n^{2}{ }_{E} n{ }_{W}\right)}{n^{3}{ }_{p}+2 n^{2}{ }_{E} n^{2}{ }_{p}+2 n^{2}{ }_{w} n^{2}{ }_{p}+3 n^{2}{ }_{E} n^{2}{ }_{w}}$

A similar treatunent can be done lo bave the other operators $P_{y} . P_{w}$ and $P_{y x}$

As described above. the finite difference method essentially translates a partial difleremial eigenualue equation into a conventional matrix eigenvalue equation. The partial differential operators have been replaced by large sparse matrices. and the eigenfunctions have been replaced by long vectors representing a sampling of the eigentunctions at discrele grid-points. Once this matrix equation was set up. one must solve for the eigemvalues and cigenvectors. Naturally: since the matrix is of dimension $M=n_{x} n_{3}$ (where $n_{x}$ and $n_{y}$ is the number of girds in $\mathrm{x}$ and $\mathrm{y}$ directions respectively), there should be $M$ eigenpairs. There are many routines available for computing a few selected eigenvalues of large sparse matrices. The most common technique is the shifted inverse power method [18]. Unfortunately, this technique proves to be relatively slow and it is only capable of computing one eigenfunction at a time. One of the most promising algorithms is the implicitly restarted Arnoldi method [19]. This method allows one to simultaneously compute a few of the largest eigenvilues of the sparse nuatrix. For this work, we used the built-in Matlab function eigs. which implements the Anoldi method.

Once the propagation constant is obtained, the chromatic dispersion D of a PCF can then be calculated from the $n_{\mathrm{err}}$ values versus the wavelength using

$\mathrm{D}=-\frac{\lambda}{\mathrm{c}} \frac{\mathrm{d}^{2} \mathrm{n}_{\mathrm{un}}}{\mathrm{d} \lambda^{2}}$

The material dispersion given by Sellmeier's formula [20] is directly included in the calculation.

The cffective mode area can also be calculated using [2I]:

$$
A_{c r s}=\frac{\left(\int_{1}^{n y, n x} \int|E(x, y)|^{2} d x d y\right)^{2}}{\int_{i}^{n y n} \int_{i}^{n y}|L(x, y)|^{4} d x d y}
$$




\section{3-Validation and numerical results}

A program implementing the Fi-l:DM method has been made. In order to check the numerical precision of the program, standard rib waveguide structure whose cross section is shown in Fig.l will be analyzed. The rib width. $W$ is $3.0 \mu \mathrm{m}$. the rib height. H. and the outer slab depth. D. are such that $H+D=1.0 \mu \mathrm{m}$. and the operating wavelength is $1.15 \mu \mathrm{m}$. The refraclive indices of the guiding, $n_{k}$ and substrate. n., regions are 3.44 and 3.4 . respectively.

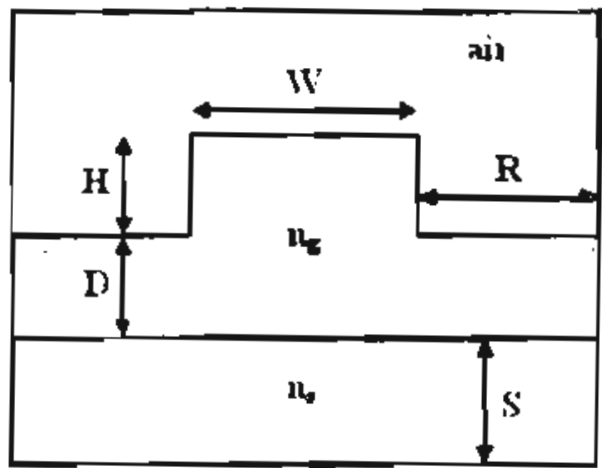

Fig. 1. Schematic diagram of the rib waveguide structure.

Table 1 shows the values of the effective index of the fundamental mode as the outer slab depth, D. varies from 0 to $0.9 \mu \mathrm{m}$. These results were obtained by using the FV-FDM and other vectorial formulations including the vector finiteelement method (VFEM), with the results from the Aitken extrapolation [22], the iterative vector finite-difference method with the transparent boundary condition (IVFDM) [23]. the semivectorial finitediflerence, method (SVFDM) [24]. the VFEM with higher order mixedinterpolation-type elements (Edge-FEM) [25] and full vectorial finite element based beam propagation method (IDVFEBPM) [26]. $\Lambda$ s may be seen from the table. there is good agreement between the results of the FV-FDM program and other formulations.

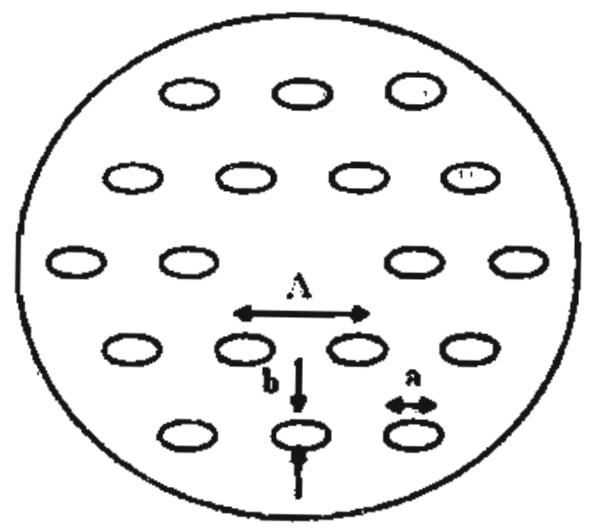

Fig. 2. Cross section of a PCF of two rings of 18 elliptical air holes. 


\begin{tabular}{|c|c|c|c|c|c|c|}
\hline$D(\mu \mathrm{m})$ & VFEM & VFDM & SV-FDM & Edge-FEM & IDVFEBPM & FV-FDM \\
\hline 0.1 & 3.41220 & $3.4121 \mathrm{I}$ & 3.41200 & 3.41209 & 3.41213 & 3.41222 \\
\hline 0.2 & 3.41235 & 3.41226 & 3.41217 & 3.41224 & 3.41229 & 3.41234 \\
\hline 0.3 & 3.41255 & 3.41247 & 3.41240 & 3.41247 & 3.41250 & 3.41252 \\
\hline 0.4 & 3.41285 & 3.41275 & 3.41271 & 3.41278 & 3.41279 & 3.41279 \\
\hline 0.5 & 3.41315 & 3.41311 & 3.41310 & 3.41312 & 3.41314 & 3.41314 \\
\hline 0.6 & 3.41365 & 3.41355 & 3.41358 & 3.41358 & 3.41358 & 3.41360 \\
\hline 0.7 & 3.41410 & 3.41408 & 3.41415 & 3.41414 & 3.41410 & 3.41415 \\
\hline 0.8 & 3.41475 & 3.41472 & 3.41484 & 3.41480 & 3.41473 & 3.41480 \\
\hline 0.9 & 3.41560 & - & 3.41568 & 3.41568 & 3.41553 & 3.41554 \\
\hline
\end{tabular}

Tablel: Effective index for the rib waveguide, shown in Fig.l. for Different values of $D$. $\mathrm{D}+\mathrm{H}=1 \mu \mathrm{m} . \mathrm{S}=1.1 \mu \mathrm{m}$ and $\mathrm{W}=3 \mu \mathrm{m}$ at $\lambda=1.15 \mu \mathrm{m}$.

Columi .2 (VFEM [22]). Column 3 (IVFDM [23]). Cohumn 4 (SV-FDM [24]). Column 5 (EdgeFEM [25]). Column 6: (IDVFEBPM [26]). Column 7: Precent work

Then the FV-FDM program has been used for pertorming modal solution for different PCirs structures. Fig. 2 shows a schematic diagram of llue PCF. consisting of two rings of arrays of elliptical air holes with $a$ and $b$ are the semiaxes of the elliptic holes (the "radii" in each direction.). They arc arranged with hole pitch $\lambda$ in a silica background whose index of relitaction taken as 1.45 at a wavelength of $1.55 \mathrm{n}: \mathrm{m}$.

For two rings of 18 air holes with $\Lambda=2 \mu \mathrm{m}$, the effect of varying a or $\mathrm{b}$ on the effective index. the dispersion and the effective mode area was tested. First a was kept constant at $0.6 \mu \mathrm{m}$ while varying $\mathrm{b}$. then keeping b constant al $0.6 \mu \mathrm{m}$ while varying a. The computing window size is $12 \mu \mathrm{m} \times 12 \mu \mathrm{m}$. For a $120 \times 120$-grid division, the computation time is 20.531 seconds for one wavelength using a Pentium IV 1.6-GHz, $256 \mathrm{MB}$. Ram personal computer. Fig.3.a. Fig.3.b and Fig.3.c slow that, the effective index, the dispersion and the eflective mode area of the lusdamental mode oblatined from the two cases are approximately identical. It may be noted that, the effective index and the effective mode area for PCF with circular holes $(a=b)$ are less than that for PCF with elliptical holes while the 
dispersion is grealer. It can also be noted thit. with increasing [de ratio (a'b) if ( $a>b)$ or increasing the ratio (b/a) if $(b>a)$, the effective index and the effective mode area increase while the dispersion decrenses. The dispersion curves become more nat and the zero dispersion wavelength is shifted to long wavelength region with increasing the above ratios. So one can reach the zero dispersion at the desired wavelenglh by controlling a and $b$ values. Therefore. if PCF with circular holes is exposed to external pressure and the holes are deformed into elliptical ones, one can expect the exposed pressure from the behavior of the effective index. the dispersion or the effective mode area.

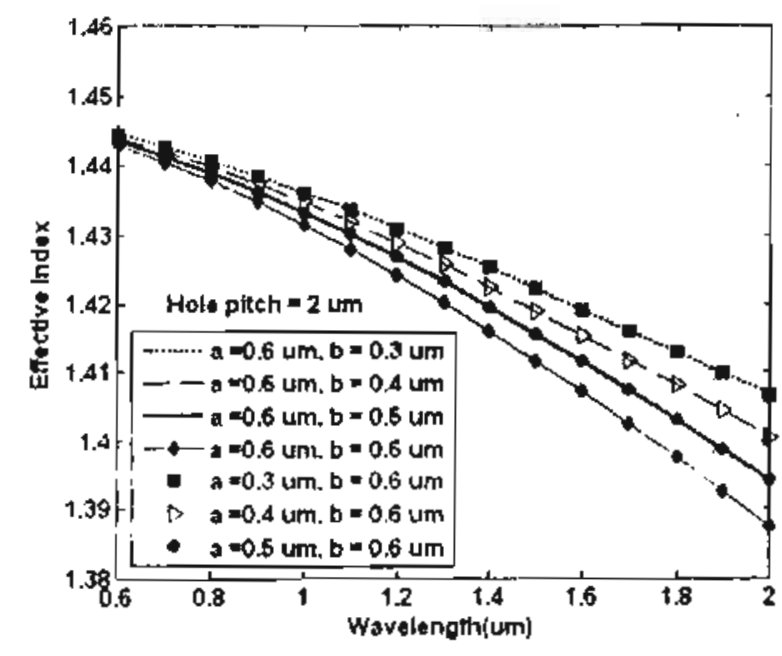

Fig.3.a Variation of effective index of the fundamental mode of two-ring PCF with elliptical holcs with waveiength with a and $b$ as parameters at $\Lambda=2 \mu \mathrm{m}$

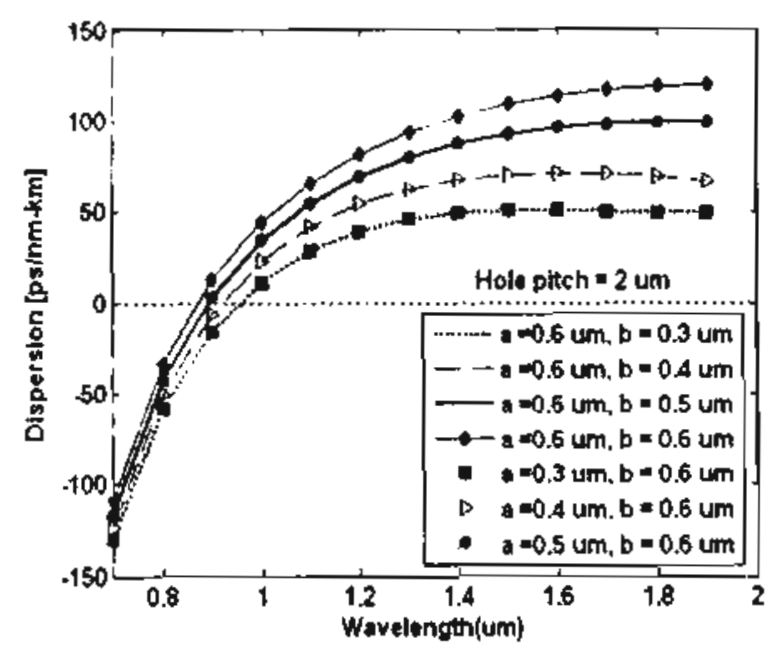

Fig.3.b Variation of dispersion of the fundamental mode of two-ring PCF with elliptical holes with wavelength with $a$ and $b$ as parameters at $\Lambda=2 \mu \mathrm{m}$ 
P. 10, M.Farhat, S.S.A.Obayya anc' A.M.Nasr

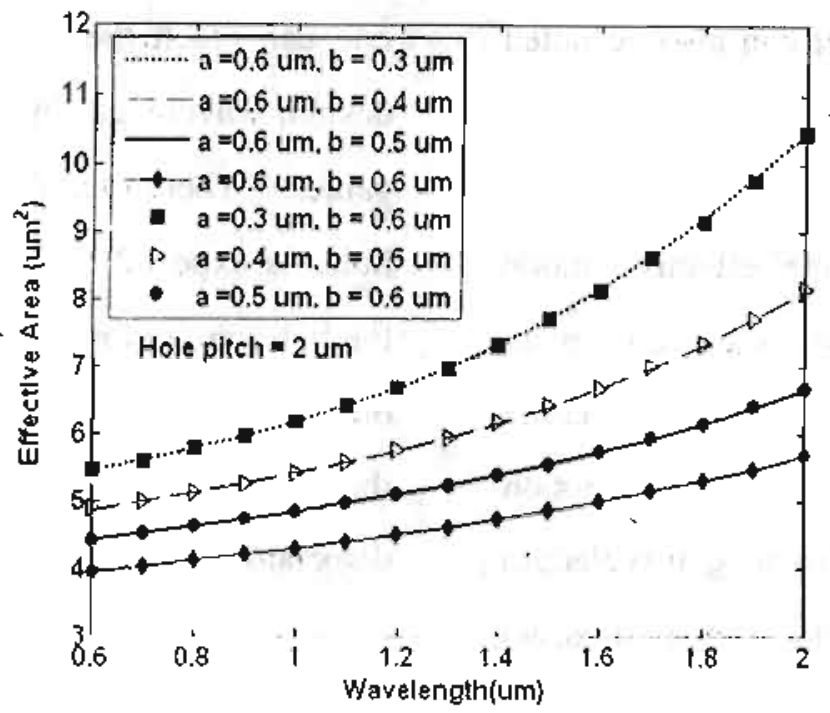

Fie.3.e Variation of elfective mode area of Ure furdamental mode ol two-ring PCF will elliptical holes with wavelength with a and b as parancters at $\Lambda=2 \mu \mathrm{m})$

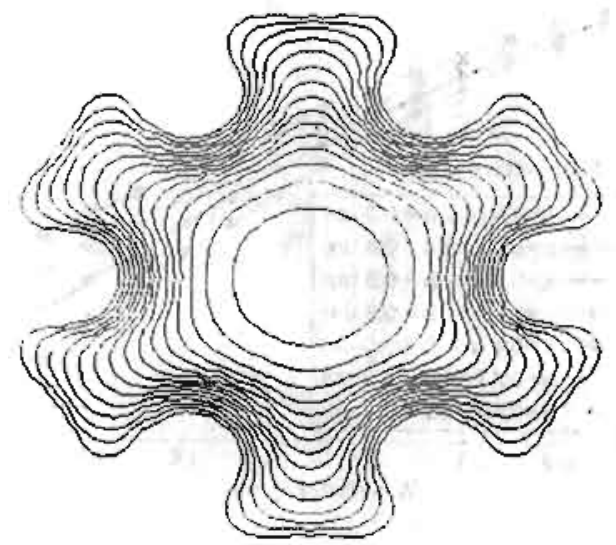

Fig.4.a The field distribution ( $\mathrm{E}_{\mathrm{v}}$ ) of the fundamental mode of two-ring $\mathrm{PCF}$ with $\Lambda=2 \mu \mathrm{m}$ and $\mathrm{a}=\mathrm{b}=0.0 \mu \mathrm{mm}$ all $\lambda=0.8 \mu \mathrm{mm}$

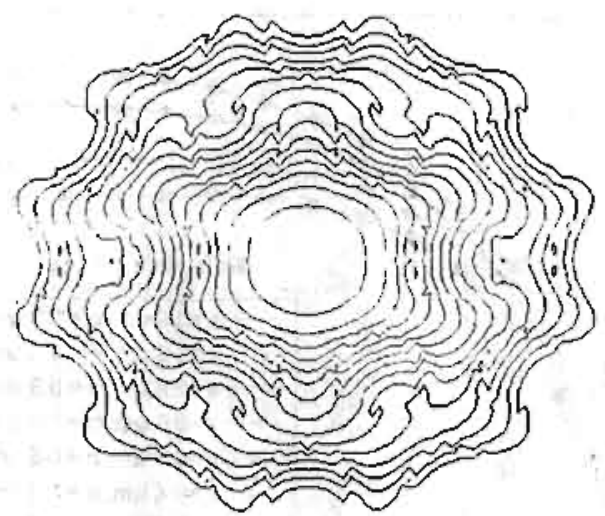

Fig.4.b The field distribution ( $E_{\mathrm{v}}$ ) of the fundamental mode of two-ring PCF with $\Lambda=2 \mu \mathrm{m}$ and $\mathrm{a}=\mathrm{b}=0.6 \mathrm{n} m \mathrm{~m}$ alt $\lambda=1.8 \mu \mathrm{m}$ 
Fig.4.a and Fig.4.b show that the mode becomes less confined to the core region with increasing the wavelength, so the effective index decreases with increasing the wavelength while the effective mode area increases as shown in Fig.3.a and Fig.3.c respectively.

Then we considered the inner ring of the PCF has circular holes while the other ring has elliptical holes. The hole radius in the tirst ring was kept constant at $0.6 \mu \mathrm{m}$ while varying a and $b$ of the elliptical logles in the outer ring. If $b$ increases while a is slifl comstam. in is noted that there is no noticeable change in the effective index as shown in Fig.5.a. However. at long wavelength the dispersion inercases while the effective mode area decreases as shown in Fig.S.b and Fins.c. The zero dispersion wavclength is not affected in this case. This ensures that the geometry of the holes in the first ring has the great effect on the effective index of the fundamental mode. So if an extemal pressure is exposed to PCF with circular holes and it deforms the holes in the second ring only into elliptic ones, then this PCF can be used as pressure sensor only at long wavelength. One can predict the pressure from the dispersion and the effective mode area curves only at long wavelength and no information can be expected in this case from the effective index curves. The abnve simulation was repeated with varying $a$ and $b$ of the elliptical holes of the outer ring but having the same area of the circular holes (that means $\left.\mathrm{a} \times \mathrm{b}=(0.6)^{2}\right)$. No significant change was obtained in the effective index. dispersion or in the effective mode area when changing the parameters of the ouler elliptical holes when they have the same area of athe first circular holes.

Keeping the area of the low index core constant, comparison between low index circular and elliptical core was made. Consider the holes of the two rings of the PCF are elliptical $(a=0.6 \mu \mathrm{m}$, $b=0.4 \mu \mathrm{m})$ and low index core with $a^{\prime} \times b^{\prime}=(0.3)^{2} \mu m^{2}$. Fig.6.a shows that there is also slight difference between the effective index of the low index circular and elliptical core when they have the same area. The dispersion and the effective mode area are shown in Fig.6.b. Fig.6.c respectively. The same results are obtained when the holes of the two rings 
of the PCF are circular. It may be noted

$1.8 \mu \mathrm{m}$ with dispersion $0 \pm 1.288$ from dispersion curves at ps/nm,km. So zero and flat dispersion can $\hat{a}^{\prime}=0.4 \mu \mathrm{m}$ and $b^{\prime}=0.225 \mu \mathrm{m}$ that the be achicved by changing the geometrica] dispersion curve is approxinately flat in parameters of the elliptical low index core the vatelongh range from 1.3 fum to $\mathrm{PCF}$ withelliptical holes

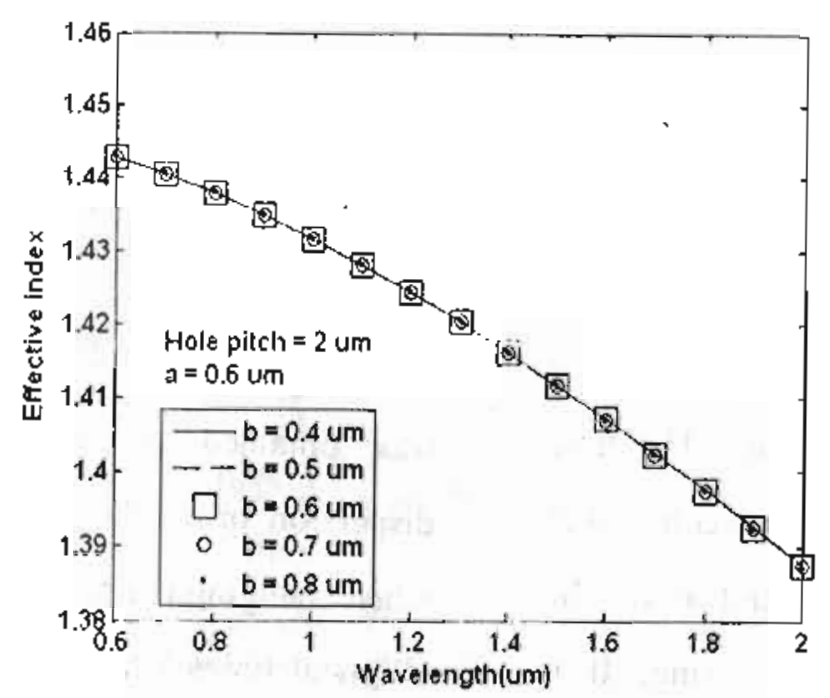

Fig.5. a Variation al erlective index of the fundamental mode of two-ring $\mathrm{PC} \%$ with wavelength with $b$ of the clliptic holes in the second ring as a parameler while keeping $(a=0.6 \mu \mathrm{m}$ ). circular holes radius in the first ring $(r=0.6 \mu \mathrm{m})$ and $(\Lambda=2 \mu \mathrm{m})$ constants

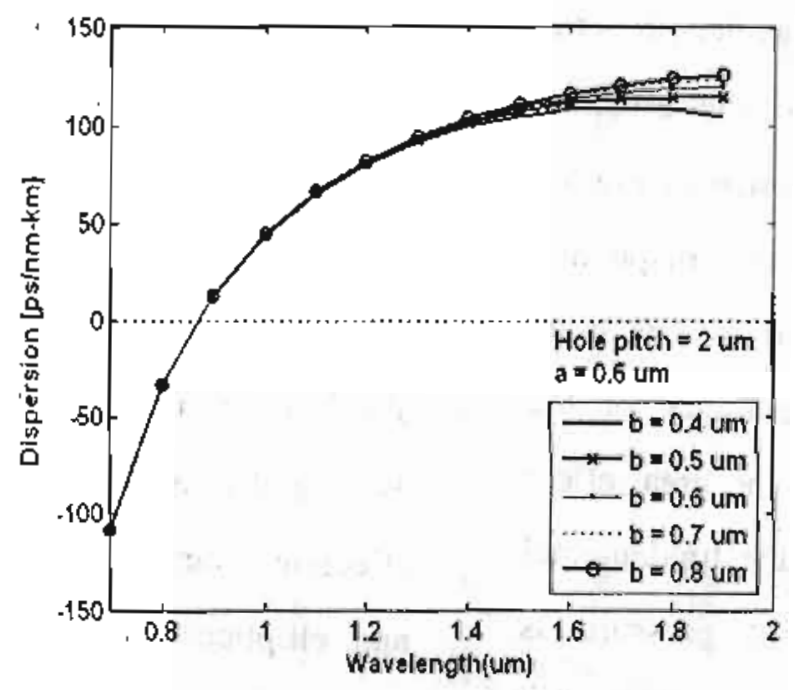

Fig.5.b Variation of dispersion of the fundamental mode of two-ring PCF with wavelength with $b$ of the elliptic holes in the second ring as a parameter while keeping $(\mathrm{a}=0.6 \mu \mathrm{m})$. circular holes radius in the first ring $(r=0.6 \mu m)$ and $(\Lambda=2 \mu \mathrm{m})$ constants 


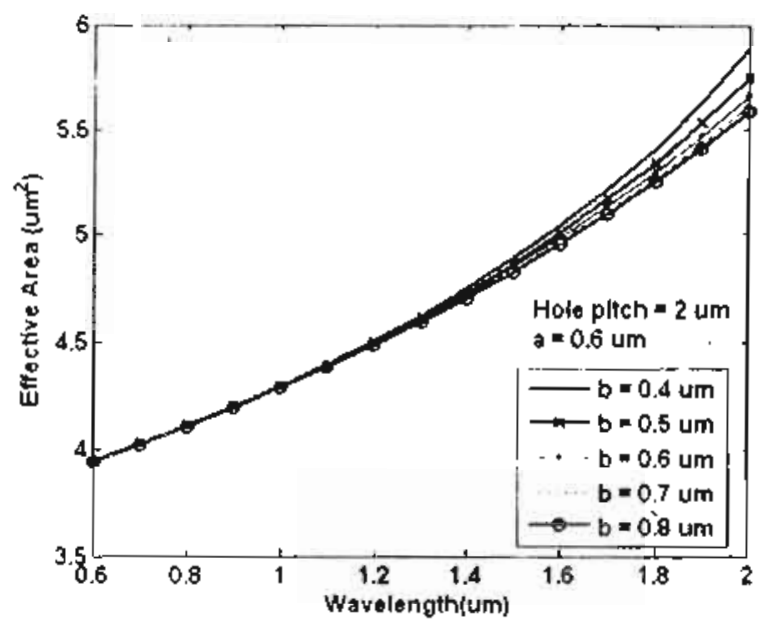

Fig.5.c Variation of effective area of the fundamental mode of two-ring PCF with wavelength with $b$ of the elliptic holes in the second ring as a parameter while keeping $(a=0.6 \mu \mathrm{m})$. circular holes radius in the first ring $(r=0.6 \mu \mathrm{m})$ and $(\Lambda=2 \mu \mathrm{m})$ constants

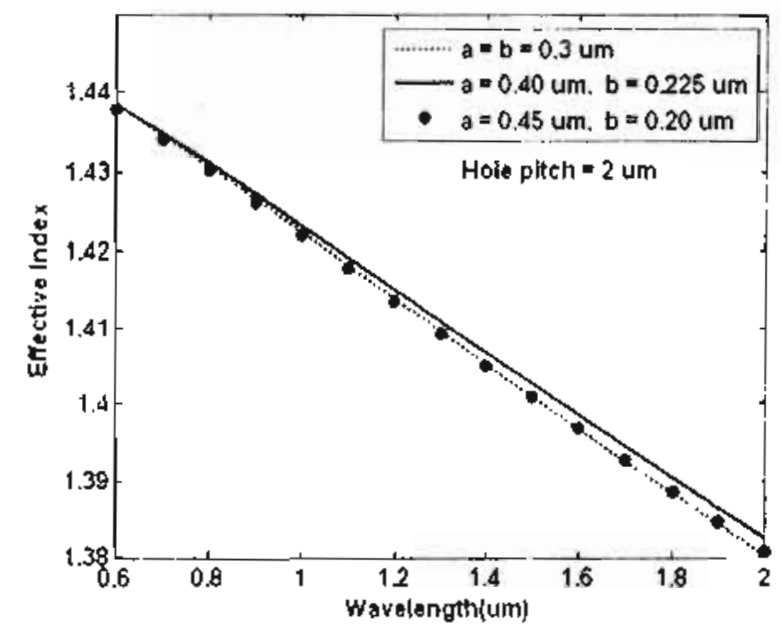

Fig.6.a Variation of effective index of the fundamental mode with wavelength with $a$ and $b$ of the central hole as parameters but having the same elliptical central hole area. The surrounding holes are elliptical.

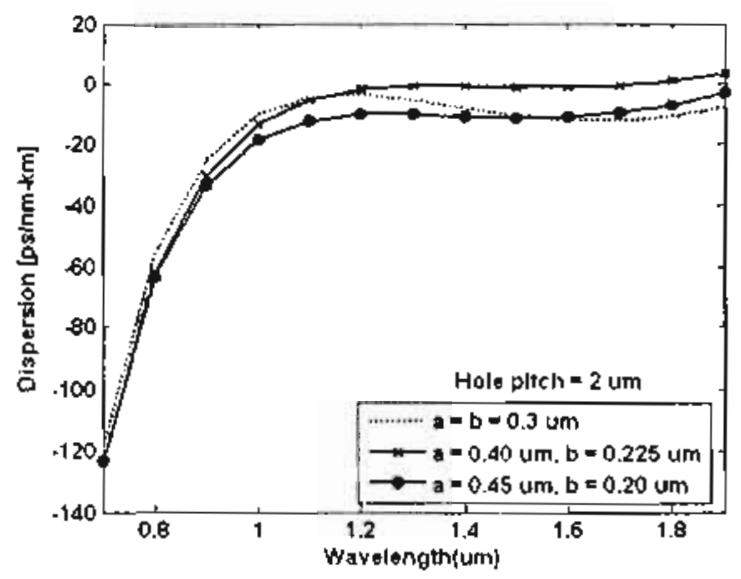

Fig.6.b Variatiun ol dispersion of the fundamental mode with wavelength with a and b of the central hole as parameters but having the same elliptical central hole area. The surrounding holes are elliptical. 


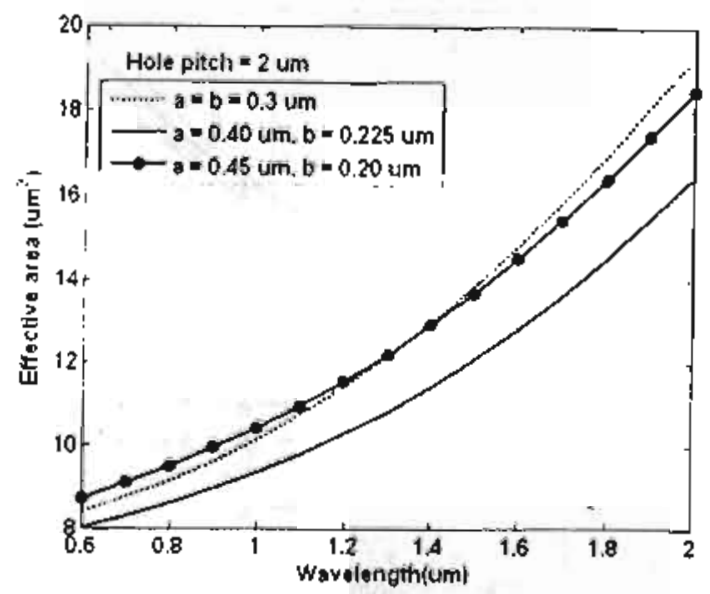

Fig.6.c Variation of effective area of the fundamental mode with wavelength with a and $b$ of the central hole as parameters bu having the same elliptical central hole area. The surrounding holes arę elliptical.

\section{4-Conclusion}

The FV-FDM has been applied to perform modal analysis of different PCFs structures wilh circular and elliptical holes. The effects of the geometrical parameters of the holes on the modal properties. such as the etleclive index. dispersion and the effective mode area are studied. 'The possibility of tailoring the PCF slructure. 10 achieve zero dispersion at the desired wavelength has been also sludied. Fial dispersion with $D=0 \pm 1.288$ $\mathrm{ps} / \mathrm{nm} . \mathrm{km}$ over the wavelength range from $1.3 \mu \mathrm{m}$ to $1.8 \mu \mathrm{m}$ has been reported. So with further optimization of the structure paramelers. these dispersions can be reduced still further. If an external pressure is exposed to PCF with circular holes and all the holes are defurmed into elliptical ones. this PCF can be used as pressure sensor. Pressure sensing can be obtained from effective index, dispersion and effective mode area curves. However, il the deformation occurs in the outer holes only, the pressure sensing can be obtained only from dispersion and the elfective mode area curves at longer wolength. No information can be expected in this casc from the effective index curves. 


\section{5- References}

[1] J.Broeng. D.Mogilevstev. S.E.Barkou. and A.Bjarklev, Photonic crystal fibers: A new class of optical waveguides, Opt. Fiber Technol. 5 (1999) 305-330.

[2] T.A.Birks, J.C.Kuight, B.J.Mangan. and P.S.J.Russell. Photonic crystal fibers: An endless variety. IF.ICF Trans. Electronm E 84-C (2001) 585-592.

[3] T.A.Birks. J.C.Knight, and P.S.J.Russell. Endlessly single-mode photonic crystat libre. Opt. Lett. 22 ( 1997) $961-963$.

[4] J.C.Knight, T.A.Birks, R.F.Cregan. P.S.J.Russell and J.P.de Sandro, Large mode area photonic crystal fiber. Electron. l.c11. 34 (1998) 1.347-1348.

[5] M.J.Gander. R.McBride. J.D.C.Jones. D.Mogilevtsev, T.A.Birks, J.C.Knight, and P.St.J. Russell, Experimental measurement of group velocity in photonic crystal liber. Electron. Lett. 35 (1998) 63-64.
[6] J.T.L.izier and G.E.Town. Splice losses in holey optical fibers, IFFF. Photonics Technol. Lett.. T3 (2001) 794-796.

[7] C.P.Yu. and H.C.Chang, Applications of the finite difference mode solution method to photonic crystal structures. Opt. Quantum Electron. 36 ( 2004) 145-163.

[8] A.Cucinotta, S.Selleri, L.Vincetti and M.Zoboli. Holey fiber analysis through the finite elentent method. IEEE Photonics Technol. Lett. 14 ( 2002) 1530-1532.

[9] M.Koshiba and K.Saitoh, Polarizationdependent continement losses in actual holey fibers. IEEE Photonics Technol. Lett. $15(2003) 691-693$.

[10] M.J.Robertson, S.Ritchie and P.Dayan, Semiconductor waveguides: analysis of optical propagation in single rib structures and directional couplers, Inst. Elec. Eng. Proc.J. 132 (1985) 336342.

1111 M.S.Stern. semivectorial polarized finite difference method for optical waveguides with arbitrary index profiles, Inst. Elec. Eng. Proc.J. 135 (1988) 56-63. 
[12] C.Vassallo. Improvement ol inite difference methods for step-index optical waveguides. Inst. Elec. Eng. Proc.J. I39 (1992) 137-142.

[13] K. Bierwirth. N.Schul\%, and F.Arndt. Finite-difference analysis of rectangular dielectric waveguides by a new finite difference method, 1. Lightwave Technol. $34(1986111041113$.

[14] P.Lusse, P.Stuwe, J.Schule and H.G.Unger. Analysis of vectorial mode fields in optical waveguides by a new finite diflerence method. I. Lightwave Technol. 12(1994) 487-494.

[15] G.L.Xu. W.P.Huang. M.S.Stem and S.K.Chaudhuri, Full-veciorial mode culculations by linite diflerence method IEE Proc.-Optoelectron. 141 (1994) 281286.

[16] A.S.Sudbo. Film Mode Matching: a Versatile Numerical Method lor Vector Mode Field Calculations in Dielectric Waveguides. Pure and Applied Optics 2 (1993) 211-233.
[17] J. Peraire. Finite Difference Discretization of Elliptic Equations. 1999. Course notes from 16.920 (Numerical Methods for Partial Dillerential Equations).

[18] M.S.Stem. Semivectorial polarised finite difference method for optical waveguides with arbitrary index profiles, IFF: Proc. II 135 (1988) 56-63

[19] D.C.Sorensen, Implicit Application of Polynomial Filters in a k-step Amoldi method. SIAM J. Matrix Analysis and Applications. 13(1) (1992) $357-385$

$$
\text { G.P.Agrawal, Fiber-optic }
$$
communication systems, (John Wiley \& Sons, 1997, 2nd Edn.). 41-42

[21] K.Saitoh and M.Koshiba, Chromatic dispersion control in photonic crystal fibers: application to ultra-flattened dispersion. Opt. Express. 11 ( 2003) 843852.

[22] B.M.A.Rahman and J.B.Davies. Vector-H finite element solution of GaAs/GaAlAs rib waveguides. Proc. Inst. 
Elect. Eng. Optoelectronics. 132 (1985) 349-353.

[23] G.R.Hadley and R.E.Smith. Fullvector waveguide modeling using :In iterative finite-difference method with transparent boundary conditions, J. Lightwave lechnol. 13 (1995) 465-469.

[24] M.S.Sterm, Semivectoria] polarized finite difference method for optical vaveguides with arbitraty index profiles. Proc. Inst. Elect. Eng. Optoelcetronics. $135(1988) 56-63$.

[25] M.Koshiba, S.Maruyama and K.Hirayanua. A vector finitc clement method with the high-order mixedinterpolation-type triangular clements for optical waveguiding problems. J.Lightwave Technol. 12 (1994) 495-502.

[26] S.S.A.Obayya. B.M.A.Rahman. K.T.V.Gratam and H.A.El-Mikall, I*ult vectorial linitc-elcment-based imagitatr distance beam propagation solution of complex modes in optical waveguides, J. Lightwave Technol. 20 ( 2002) 10541060 . 